\title{
A spectroscopic ruler for intermediate-zone FRET measurements
}

\author{
Garth A. Jones* and David L. Andrews \\ School of Chemistry, University of East Anglia, Norwich Research Park, Norwich, Norfolk, NR4 \\ 7TJ, United Kingdom.
}

\begin{abstract}
It is well known that Fluorescence Resonance Energy Transfer (FRET), the most common mechanism for electronic energy to migrate between molecular chromophores, has a predominantly inverse sixth power dependence on the rate of transfer as a function of the distance $R$ between the chromophores. However, the unified theory of electronic energy transfer, derived from quantum electrodynamics, predicts an additional contribution with an $R^{-4}$ dependence on distance. This intermediate-zone term becomes especially important when the chromophore spacing is similar in magnitude to the reduced wavelength $(\lambda=\lambda / 2 \pi)$ associated with the mediated energy. In previous theoretical studies we have suggested that inclusion of the intermediate term, through rate equation and quantum dynamical calculations, may be important for describing the exciton diffusion process in some circumstances, and in particular when the distance between the chromophores exceeds $5 \mathrm{~nm}$. In this paper, we focus of the role of the intermediate-zone contribution to distance measurements between chromophores made through the application of spectroscopic ruler techniques. One of the major assumptions made in employing these experimental techniques is that the $R^{-6}$ dependence is valid. In this work, we reformulate the spectroscopic ruler principles for intermediate distances to include the inverse fourth power rate component, and compare the results of this reformulation to experimental FRET results from the literature.
\end{abstract}

Keywords: FRET, spectroscopic ruler, electronic energy transfer, virtual photon, dipole approximation, quantum electrodynamics

*garth.jones@uea.ac.uk; phone +44 160359 1469; fax +44 1603592003

\section{INTRODUCTION}

\subsection{Background}

Fluorescence resonance energy transfer (FRET) is the process by which an electronically excited state in one molecular center - often a characteristic group or extrinsic dopant ion - is given up to create an excited state in another nearby molecular center or chromophore. ${ }^{1}$ This is process is fundamental to the initial stage of photosynthesis and at the basic level occurs via the exchange of a photon.

When the species involved in the energy transfer process are relatively close together, the photon has a 'virtual' character. That is to say, the mediating photon has special near-zone or quantum uncertainty traits when its reduced wavelength $(\lambda=\lambda / 2 \pi)$ is large, in comparison to the distance of separation between the chromophores. Under these circumstances, the photon is only in existence for a very short time and cannot take on any real character. The associated type of energy transfer is phenomenologically radiationless and is referred to as near-zone energy transfer. This is the type of transfer has rate constants that run with $1 / R^{6}$, where $R$ is the distance separating the chromophores. FRET models assume this type of EET, which is often referred to as near-zone. In the other extreme, when the two molecules are very far apart, such that the reduced wavelength is much smaller than the inter-chromophore distance, the EET process is called radiative, consistent with the fact that the mediating photon has real character and is therefore experimentally observable. The rate of EET now has the 'Coulombic' $1 / R^{2}$ distance dependence and is referred to as far-zone. These two processes were once thought to be independent mechanisms, however it is now known that they represent two limits of a unified theory of EET, ${ }^{2,3}$

Ultrafast Phenomena and Nanophotonics XIX, edited by Markus Betz, Abdulhakem Y. Elezzabi,

Kong-Thon Tsen, Proc. of SPIE Vol. 9361, 93610I - (c) 2015 SPIE

CCC code: $0277-786 \mathrm{X} / 15 / \$ 18 \cdot$ doi: $10.1117 / 12.2076913$

Proc. of SPIE Vol. 9361 93610l-1 
The Unified Theory of EET was derived from quantum electrodynamics (QED), and very interestingly also predicted that when the reduced wavelength is of the order of the distance between the donor and the acceptor an intermediatezone of energy transfer exists that has a $1 / R^{4}$ dependence on the rate of EET. We have recently published two papers, one investigating electronic coupling and rate constants, ${ }^{4}$ and the other quantum dynamics, ${ }^{5}$ indicating that the $1 / R^{4}$ term may be non-negligible for chromophores separated by relatively short distances (typically $5 \mathrm{~nm}$ or greater).

In 1967, Stryer and Haugland proved experimentally that there is a $1 / R^{6}$ dependence of the efficiency of EET. ${ }^{6}$ In this work they coined the term 'spectroscopic ruler', and since then the technique has been applied to a huge number of applications for the purpose of measuring the distance between to photo-active molecular centers. We present the basics of the theory in Section 2.2. Although the spectroscopic ruler technique is very powerful, there have been a number of recent studies that have observed departures from the $1 / R^{6}$ trend. These are typically studies that consider the EET process over distances significantly greater than several nanometers. ${ }^{7-10}$ These, and other, studies have motivated us to initiate a project that seeks to recalibrate the spectroscopic ruler for distances at exceeding the near-zone, where departures from $1 / R^{6}$ take place. The underlying theory upon which this recalibration will take place is that of quantum electrodynamics (QED).

\section{BACKGROUND THEORY}

\subsection{QED Theory of EET}

FRET is a quantum mechanical process and therefore when describing it theoretically, one starts from the Hamiltonian describing the system. For simplicity, the case of a two-chromophore system will be considered where electronic energy transfer occurs from the donor to the acceptor chromophore. Excluding, for simplicity, the modifying effects of a surrounding medium, the system can be described by the Hamiltonian,

$$
H=H_{\text {mol }}(D)+H_{\text {mol }}(A)+H_{\text {rad }}+H_{\text {int }}(A)+H_{\text {int }}(D)
$$

where $H_{\text {rad }}$ describes Hamiltonian for the radiation field, $H_{\text {mol }}(X)$ is the usual molecular Hamiltonian for species $X(=A$, $D$ respectively signifying the energy donor and acceptor) positioned at $\mathbf{R}_{X}$. Full details of the components of the Hamiltonians can be found elsewhere. ${ }^{11}$ The electronic coupling between chromophores occurs strictly through the interaction of the molecular sub-systems and the quantized field, i.e. there is no term in the Hamiltonian that directly couples the two chromophores (in contrast to in semiclassical treatments). The dipole interaction Hamiltonian is given by

$$
H_{\text {int }}(X)=-\varepsilon_{0}^{-1} \vec{\mu}_{\alpha}(X) \cdot \hat{d}_{\alpha}^{\perp}\left(\mathbf{R}_{X}\right)
$$

where $\vec{\mu}_{\alpha}(X)$ is the transition dipole moment between the electronic ground state and state $\alpha$ of chromophore $X$, and $\hat{d}_{\alpha}^{\perp}\left(\mathbf{R}_{X}\right)$ is the electric displacement field operator which relates to the mediating photon. Following standard QED (quantum electrodynamics) procedures, using this Hamiltonian to calculate the matrix element connecting the initial and final states, one arrives at the EET rate equation through the Fermi Golden Rule:

$$
W_{F I}=\frac{2 \pi}{\hbar}|\langle F|\hat{T}| I\rangle|^{2} \delta\left(E_{I}-E_{F}\right)
$$

Here $\hat{T}$ is the transition operator (taken to second order) and the wavefunctions are written formally in terms of initial and final states. Taking the mathematical derivation further leads us to the rate equation, which is written in terms of overlap integrals between the donor and acceptor spectra. Writing the circular frequency as $\omega=c k$ gives,

$$
W_{D A}=\frac{9}{8 \pi c^{2} \tau_{D}} \int_{0}^{\infty} F_{D}(\omega) \sigma_{A}(\omega) g(\omega ; \mathbf{R}) d \omega
$$


with

$$
g(\omega, \mathbf{R})=\eta_{3}^{2} \frac{c^{6}}{\omega^{6} R^{6}}+\left(\eta_{3}^{2}-2 \eta_{1} \eta_{3}\right) \frac{c^{4}}{\omega^{4} R^{4}}+\eta_{1}^{2} \frac{c^{2}}{\omega^{2} R^{2}}
$$

The functions $\sigma_{A}(\omega)$ and $F_{D}(\omega)$ are the absorption cross-section of the acceptor and the emission spectrum of the donor, respectively, and the parameter $\tau_{D}$ is the radiative lifetime of the donor. Full details of these terms can be found in chapter 2 of reference 11 . The parameter $g(\omega, \mathbf{R})$ is analogous to the electronic coupling. The three different regimes of EET can be clearly seen in equation (5), namely the near-zone, $1 / R^{6}$ term, the intermediate-zone, $1 / R^{4}$ term and the farzone, $1 / R^{2}$ term. Note that only in the case of EET occurring in highly absorbing media do $1 / R^{3}$ and $1 / R^{5}$ terms appear. These are not considered here, but details can be found in chapter 2 of reference 11 . The orientational factors $\eta_{q}(q=1,3)$, describe the influence of the relative orientations of the transition dipole moments of the donor and acceptor chromophores, given by

$$
\eta_{q}=\left(\hat{\vec{\mu}}_{D} \cdot \hat{\vec{\mu}}_{A}\right)-q\left(\hat{\vec{R}} \cdot \hat{\vec{\mu}}_{A}\right)\left(\hat{\vec{R}} \cdot \hat{\vec{\mu}}_{D}\right)
$$

In the case of near- and intermediate-zone coupling, for which $q=3$, this factor becomes most favorable when the transition dipole moments are parallel (or anti-parallel) to one another and to the displacement vector. In the case of farzone couplings, where $q=1$, the orientation factor is most favorable when the transition dipole moments are parallel to one another, but perpendicular to the displacement vector.

\subsection{The Spectroscopic Ruler}

In this subsection, the basic theory behind the usual $1 / R^{6}$ dependent FRET and the spectroscopic ruler is discussed. FRET conveniently occurs with highest efficiencies over the range of distances associated with macromolecular internal dimensions, and measurements based on this technique have been extensively used as a spectroscopic ruler for probing distances between chromophores over distances that range from $2-6 \mathrm{~nm}^{7,12-13}$ These measurements are based on the comparing the efficiency of energy transfer against known standards. The well-known expression for the rate of electronic energy transfer between a donor and acceptor, separated by distance $R$, and based on this inverse sixth power law, is given by

$$
k_{E E T}(r)=\frac{Q_{D} \kappa^{2}}{\tau_{D} R^{6}}\left(\frac{9000(\ln 10)}{128 \pi^{5} N n^{4}}\right) \int_{0}^{\infty} F_{D}(\lambda) \varepsilon_{A}(\lambda) \lambda^{4} \mathrm{~d} \lambda
$$

where $\tau_{D}$ is the lifetime of the donor in the absence of the acceptor, $Q_{D}$ is the quantum yield of the donor, $\kappa^{2}$ is the square of the orientational factor, $F_{D}(\lambda)$ is the (corrected) fluorescence intensity of the donor in the range $\lambda+d \lambda$ and $\varepsilon_{a}(\lambda)$ is the extinction coefficient.

For practical reasons, in the case of spectroscopic ruler techniques, the rate of energy transfer is commonly defined in terms of the Förster distance $R_{0}$. This is the distance between the chromophores when the transfer rate $k_{E E T}(r)$ is equal to the decay rate of the donor in the absence of the acceptor $\tau_{D}^{-1}$. That is, when the two molecules are separated by $R_{0}$, exactly half of the molecules will decay by EET and half will decay via radiative and non-radiative mechanisms. The rate equation then becomes

$$
k_{E E T}(r)=\frac{1}{\tau_{D}}\left(\frac{R_{0}}{R}\right)^{6}
$$

with

$$
R_{0}^{6}=\frac{9000(\ln 10) \kappa^{2} Q_{D}}{128 \pi^{5} N n^{4}} \int_{0}^{\infty} F_{D}(\lambda) \varepsilon_{A}(\lambda) \lambda^{4} \mathrm{~d} \lambda
$$


The expression for $R_{0}$ can be calculated from the spectral properties of the donor and acceptor (as individual species) and from the donor quantum yield.

Having established how these mechanisms compete kinetically, one can now define the problem in terms of efficiencies, by measuring the amount of photons absorbed by the donor that are then subsequently transferred to the acceptor. This fraction (i.e. the efficiency) is given by

$$
E=\frac{k_{E E T}}{\tau_{D}^{-1}+k_{E E T}}
$$

Combining equations (8) and (10) results in expression (11)

$$
E=\frac{R_{0}^{6}}{R_{0}^{6}+R^{6}}
$$

It can be that the transfer efficiency is strongly dependent $r$ near $\mathrm{R}_{0}$. The transfer efficiency can be more practically measured using relative fluorescence intensity of the donor in the absence (FD) and presence (FD-A) of the acceptor as well as the lifetimes under these respective conditions

$$
E=1-\frac{\tau_{D A}}{\tau_{D}}
$$

and

$$
E=1-\frac{F_{D A}}{F_{D}}
$$

\section{AN INTERMEDIATE-ZONE SPECTROSCOPIC RULER}

\subsection{Spectroscopic ruler for intermediate zone}

The spectroscopic ruler approach described above assumes that there are only two possible mechanisms by which the excited state of the donor molecule can be deactivated; namely via radiationless EET that has an $1 / R^{6}$ dependence on separation between the donor and acceptor, or radiative or non-radiative decay via direct means, such as fluorescence or heat. In many applications this is a completely valid assumption. In particular, when the donor and acceptor species are much closer together than the reduced wavelength associated with the transferred energy. In cases where the distance between the donor and acceptor becomes non-negligible compared to this reduced wavelength, radiative energy transfer effects start to play a decisive role in the EET dynamics. At distances approaching this reduced wavelength, radiative effects actually start to dominate. To put this in perspective, for UV radiation, the photons associated with this energy will have a reduced wavelength $30-60 \mathrm{~nm}$. One can therefore expect that donor - acceptor distances that are a significant fraction of this dimension will have significant contributions from the intermediate- and far- zone terms appearing in equation (8). In fact, in two recent studies undertaken by us and co-workers, we have found that deviations from Förster theory occurs in the electronic couplings, kinetics and quantum dynamics when compared to QED calculations for as little as $5 \mathrm{~nm}$.

In this work the spectroscopic ruler expressions are derived to include both intermediate- and far-zones terms. We begin by deconstructing the rate equation derived from QED into its three fundamental contributions, the near-, intermediateand far-zone rates, respectively. To avoid confusion with Förster theory rate constants, we label the QED-derived rate constants $W_{D A}$, with subscripts indicating specific contributions. Equating equation (4) to equation (11) for the near-zone $R^{-6}$ contribution only in equation (5) only, 


$$
W_{D A}^{\left(R^{-6}\right)}=\frac{9 c^{4} \eta_{3}^{2}}{8 \pi c^{2} \tau_{D} R^{6}} \int_{0}^{\infty} F_{D}(\omega) \sigma_{A}(\omega) \omega^{-4} d \omega=\frac{1}{\tau_{D}}\left(\frac{R_{0}}{R}\right)^{6}
$$

We can now express a new QED rate equation in terms of the Förster radius that incorporates all three distance dependences. In is important to realize that intermediate- and far-zone contributions to the EET rate have different orientational factors than the near-zone. This is due fundamentally to the changing character of the oscillating fields associated with the photon as it moves away from the donor (see reference 4, and references therein for details). Consequently, when deriving the contributions to the rate equations from the $R^{-4}$ and $R^{-2}$ terms, these factors must be taken into account. When the rate equation is written in terms of the Förster radius, the orientation factor is commonly taken to be the rotationally averaged value, $\eta_{3}^{2}=2 / 3$. This is accounted for in the following expression for the rate equation, where the chromophores are no longer assumed to be orientationally averaged (the superscript tilde, $\sim$, defines the rate constant for a non - orientationally averaged rate constant)

$$
\tilde{W}_{D A}^{\left(R^{-6}\right)}(R)=\left(\frac{3}{2}\right)^{\frac{1}{6}} \frac{\eta_{3}^{2}}{\tau_{D}}\left(\frac{R_{0}^{6}}{R^{6}}\right)
$$

The intermediate- and far-zone contributions to the EET rate then follow;

$$
\tilde{W}_{D A}^{\left(R^{-4}\right)}(R)=\left(\frac{3}{2}\right)^{\frac{1}{6}} \frac{k^{2}}{\tau_{D}}\left(\eta_{1}^{2}-2 \eta_{1} \eta_{3}\right)\left(\frac{R_{0}^{6}}{R^{4}}\right)
$$

and

$$
\tilde{W}_{D A}^{\left(R^{-2}\right)}(R)=\left(\frac{3}{2}\right)^{\frac{1}{6}} \frac{k^{4}}{\tau_{D}} \eta_{1}^{2}\left(\frac{R_{0}^{6}}{R^{2}}\right)
$$

The total rate equation, including all three terms, is now written in final form as,

$$
\tilde{W}_{D A}^{\text {Full }}(r)=\left(\frac{3}{2}\right)^{\frac{1}{6}} \frac{R_{0}^{6}}{\tau_{D}}\left(\frac{\eta_{3}^{2}}{R^{6}}+\frac{k^{2}\left[\eta_{3}^{2}-2 \eta_{1} \eta_{3}\right]}{R^{4}}+\frac{k^{4} \eta_{1}^{2}}{R^{2}}\right)
$$

If the relative orientations of the chromophores are known, this information may be inserted into the new rate equation (18) using equation (9). However, orientational averaging factors of $\eta_{1}^{2}=1 / 9, \eta_{1} \eta_{3}=0$ and $\eta_{3}^{2}=2 / 3$ can be input for isotropic cases. Note that $k$ is the wave-number of the mediated energy. Inserting equation (18) to equation (10), where $k_{E E T}(R)=\tilde{W}_{D A}^{F u l l}(R)$, the following expression for efficiency is obtained: 


$$
E_{\text {QED }}=\frac{\tilde{W}_{D A}^{\text {Full }}}{1+\tilde{W}_{D A}^{F u l l}}=\frac{1}{\left(\frac{3}{2}\right)^{\frac{1}{6}} R_{0}^{-6}\left(\frac{\eta_{3}^{2}}{R^{6}}+\frac{k^{2}\left[\eta_{3}^{2}-2 \eta_{1} \eta_{3}\right]}{R^{4}}+\frac{k^{4} \eta_{1}^{2}}{R^{2}}\right)^{-1}}
$$

Although this expression is somewhat more unwieldy than equation (11), the efficiency expression now includes contributions from the radiative contributions to the EET process. The only additional piece of information required to calculate the efficiency, $E$, as a function of $\left(R / R_{0}\right)$ is the wave-number for the transferred energy, which can be identified from the fluorescence spectrum of the energy transfer donor.

\subsection{Comparing Förster Theory with QED (including intermediate- and far-zone terms)}

Here we present a sample qualitative calculation comparing the efficiency of EET derived from Förster theory and the full quantum electrodynamical treatment. Here we have assumed for illustrative purposes that the orientational factors correspond to the rotationally averaged case and that $k=1$.

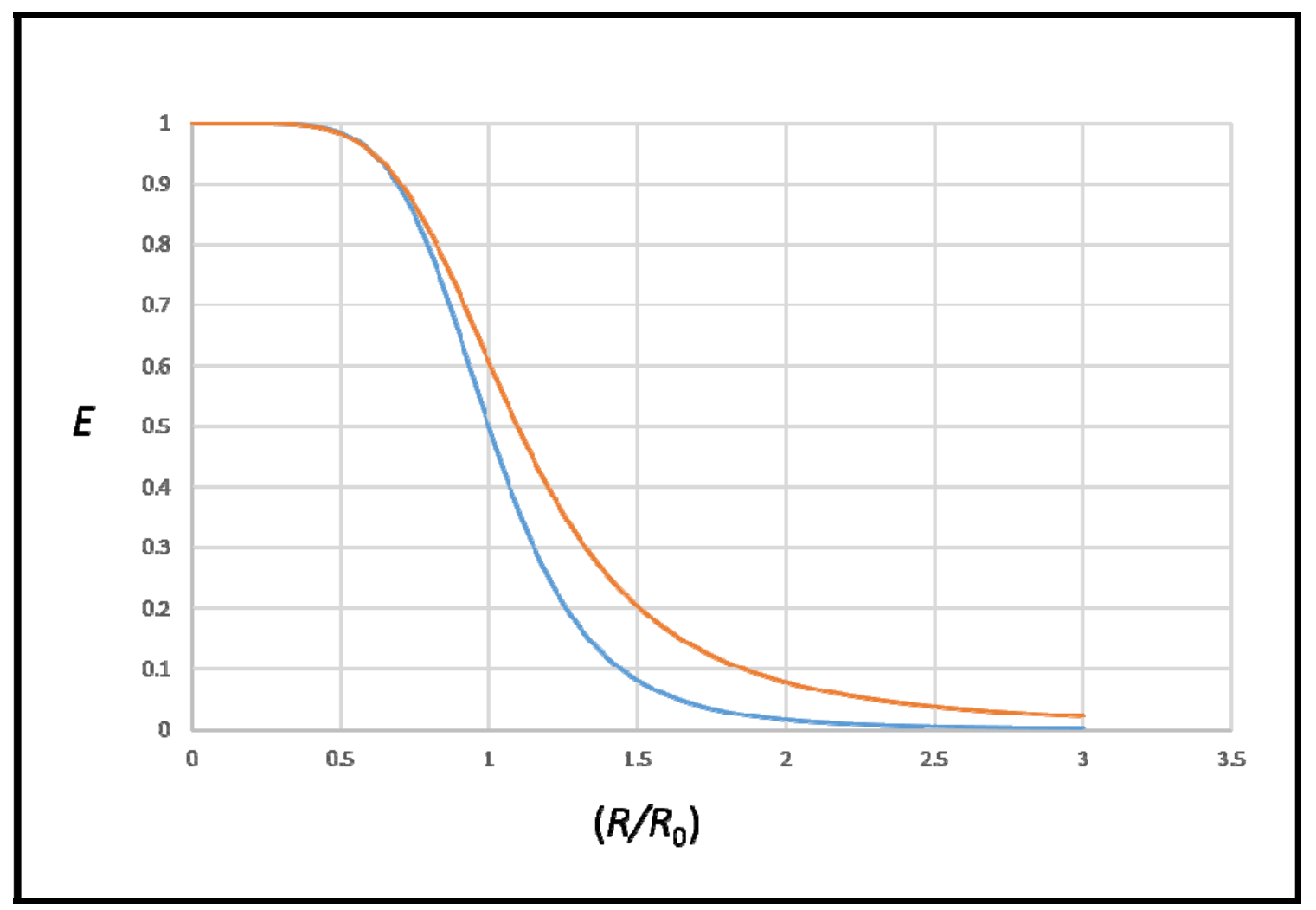

Figure 1. A comparison of the efficiency of EET for rates of Förster theory (blue/lower) and QED (orange/upper). The Försterderived curve is calculated using equation (11), and the QED efficiency from the derived equation (19).

Looking at the efficiency curves of Figure 1, it can be seen that the intermediate- and far-zone terms play a significant role in increasing efficiency at large values of $\left(R / R_{0}\right)$. In fact, the more precise QED formulation suggests efficiencies that are larger by a factor of more than 2 , when the inter-chromophore distances are of the order of the Förster radius. Distance measurements based on these two theories are in consequence expected to differ by approximately $15 \%$. 


\section{OUTLOOK}

In this paper, we have undertaken initial calculations that should lead to a re-evaluation of the spectroscopic rule for measuring distances between molecular centers that are significantly further apart than the Förster radius. The development of such an approach should lead to predictions of correct EET behavior in the intermediate-zone, the more accurate theory leading to results that can be seen to depart significantly from those results associated with the simpler

Förster theory. These results would then conform to several recent studies that have observed non-FRET behavior over large distances. ${ }^{7,8,10}$

\section{REFERENCES}

[1] Förster, Zwischenmolekulare Energiewanderung und Fluoreszenz, T. Ann. Phys. (Leipzig) 2, 55 (1948).

[2] Andrews, D. L., A unified theory of radiative and radiationless molecular energy transfer, Chem. Phys. 135, 195 (1989).

[3] Andrews, D. L., Bradshaw, D. S., Virtual photons, dipole fields and energy transfer: a quantum electrodynamical approach, Eur. J. Phys. 25, 845 (2004).

[4] Lock, M. P. E, Andrews, D. L., Jones G. A., On the nature of long range electronic coupling in a medium: Distance and orientational dependence for chromophores in molecular aggregates, J. Chem. Phys., 140, 044103 (2014).

[5] Frost J. E., Jones, G. A., A quantum dynamical comparison of the electronic couplings derived from quantum electrodynamics and Förster theory: application to 2D molecular aggregates, New J. Phys., 16, 113067 (2014).

[6] Stryer, L., Haugland, R. P., Energy transfer: a spectroscopic ruler, Proc. Natl. Acad. Sci. U.S.A., 58, 179, (1967).

[7] Sen, T., Sadhu, S., Patra, A., Surface energy transfer from rhodamine 6G to gold nanoparticles: A spectroscopic ruler, Appl. Phys. Lett. 91, 043104 (2007).

[8] Swathi, R. S., and K. L. Sebastian, Resonance energy transfer from a fluorescent dye molecule to plasmon and electron-hole excitations of a metal nanoparticle, J. Chem. Phys. 126, 234701 (2007).

[9] Koushik, S. V., P. S. Blank, and S. S. Vogel, Anomalous surplus energy transfer observed with multiple FRET acceptors, PloS ONE 4, e8031 (2009).

[10] Chatterjee, S., Lee, J. B., Valappil, N. V., Luo, D., and Menon, V. M., Investigating the distance limit of a metal nanoparticle based spectroscopic ruler, Biomed. Opt. Express 2, 1727-1733 (2011).

[11] Andrews, D. L., Demidov, A. A. Resonance Energy Transfer (Wiley, Chichester, 1999).

[12] Stryer, L.Ann. Rev. Bio. Chem., Fluorescence energy transfer as a spectroscopic ruler, 47, 819 - 846 (1978).

[13] Schuler, B. , Lipman, E. A., Steinbach, P.J., Kumkell, M., Eaton, W.A., Polyproline and the "spectroscopic ruler" revisited with single-molecule fluorescence, Proc. Natl. Acad. Sci. U.S.A, 102, 2754 (2005). 